\title{
Use of 3-dimensionally printed heart models in the planning and simulation of surgery in patients with Raghib syndrome (coronary sinus defect with left superior vena cava)
}

\author{
Nabil Hussein, MBChB (Hons), ${ }^{\text {a,b }}$ Reda Kasdi, MD, ${ }^{\mathrm{a}, \mathrm{b}}$ John G. Coles, MD, ${ }^{\mathrm{a}, \mathrm{b}}$ and Shi-Joon Yoo, MD ${ }^{\mathrm{a}, \mathrm{c}}$
}

\footnotetext{
From the ${ }^{\mathrm{a}}$ Division of Cardiology, Department of Paediatrics, ${ }^{\mathrm{b}}$ Division of Cardiovascular Surgery, Department of Surgery, and ${ }^{\mathrm{c}}$ Department of Diagnostic Imaging, Hospital for Sick Children, University of Toronto, Toronto, Ontario, Canada.

Disclosures: The authors reported no conflicts of interest.

The Journal policy requires editors and reviewers to disclose conflicts of interest and to decline handling or reviewing manuscripts for which they may have a conflict of interest. The editors and reviewers of this article have no conflicts of interest.

Received for publication Dec 3, 2019; revisions received Dec 3, 2019; accepted for publication Jan 8, 2020; available ahead of print Feb 23, 2020.

Address for reprints: Shi-Joon Yoo, MD, Division of Cardiology, Department of Paediatrics, Department of Diagnostic Imaging, Hospital for Sick Children, University of Toronto, 555 University Ave, Toronto, Ontario M5G 1X8, Canada (E-mail: shi-joon.yoo@sickkids.ca).

JTCVS Techniques 2020;2:135-8

2666-2507

Crown Copyright $\Subset 2020$ Published by Elsevier Inc. on behalf of The American Association for Thoracic Surgery. This is an open access article under the CC BY-NC-ND license (http://creativecommons.org/licenses/by-nc-nd/ $4.0 /)$.

https://doi.org/10.1016/j.xjtc.2020.01.023
}

Raghib syndrome, also known as coronary sinus defect with persistent left superior vena cava (LSVC), consists of complete unroofing of the partial wall between the coronary sinus and the left atrium in the presence of persistent LSVC. ${ }^{1}$ It is characterized hemodynamically by drainage of the LSVC to the left atrium and a large interatrial shunt through the ostium of the unroofed coronary sinus. This article describes the use of 3-dimensional (3D) models in the presurgical planning and simulation of 2 patients with Raghib syndrome.

\section{PATIENTS AND METHODS \\ Patient Profiles}

Two patients ( 5 years, $17.6 \mathrm{~kg}$; and 6 years, $16.2 \mathrm{~kg}$ ) were referred for elective repair of Raghib syndrome. Cross-sectional imaging was performed to depict the patients' anatomy accurately. From these data, 3D-printed models were produced to assist with anatomy interpretation and simulate the technical approaches before the operation (Figure 1). Our Research Ethics Board does not require the patient's consent because there is no identifiable personal health information.

\section{Surgical Techniques Used and Outcomes}

The models were printed with Agilus clear resin (Stratsys Ltd, Eden Prairie, Minn), a soft material suitable for simulation. Both intracardiac and extracardiac repairs were evaluated for feasibility on the models by assessing the anatomic data.

Data assessed for intracardiac repair were as follows:

1. Pulmonary vein orifices location and proximity to the LSVC orifice.

2. Required length for an intra-atrial baffle along the posterior aspect of the mitral valve annulus. (RSVC). (RAA). RAA.

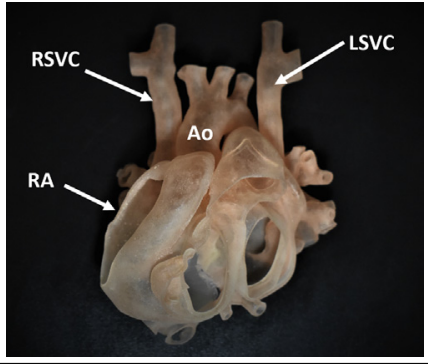

3D-printed model of patient with Raghib syndrome for surgical planning and simulation.

\section{CENTRAL MESSAGE \\ Three-dimensionally printed models can be used successfully in the preoperative decision making for patients with complex congenital heart defects.}

See Commentaries on pages 139, 141, and 143

3. Required length of an intra-atrial baffle along the posterior wall of the left atrium above the pulmonary vein orifices.

Data assessed for extracardiac repair were as follows:

1. Length of the dissectible part of the LSVC.

2. Distance between the upper LSVC and lower right superior vena cava

3. Distance between the upper LSVC and the right atrial appendage

4. Distance between the ascending aorta and the sternum.

Surgical options included are as demonstrated in Figure 2:

1. Division of the LSVC at its orifice and anastomosing it to the RSVC or

2. Interposition conduit between the LSVC and the RSVC or RAA.

3. Intra-atrial baffle along the roof or floor of the left atrium from the LSVC orifice to the right atrium.

Possible challenges of the techniques were considered, and all the methods were simulated on the models. An intra-atrial baffle along the roof of the left atrium was deemed the best option for both cases. It would be least likely to affect the pulmonary venous drainage and 

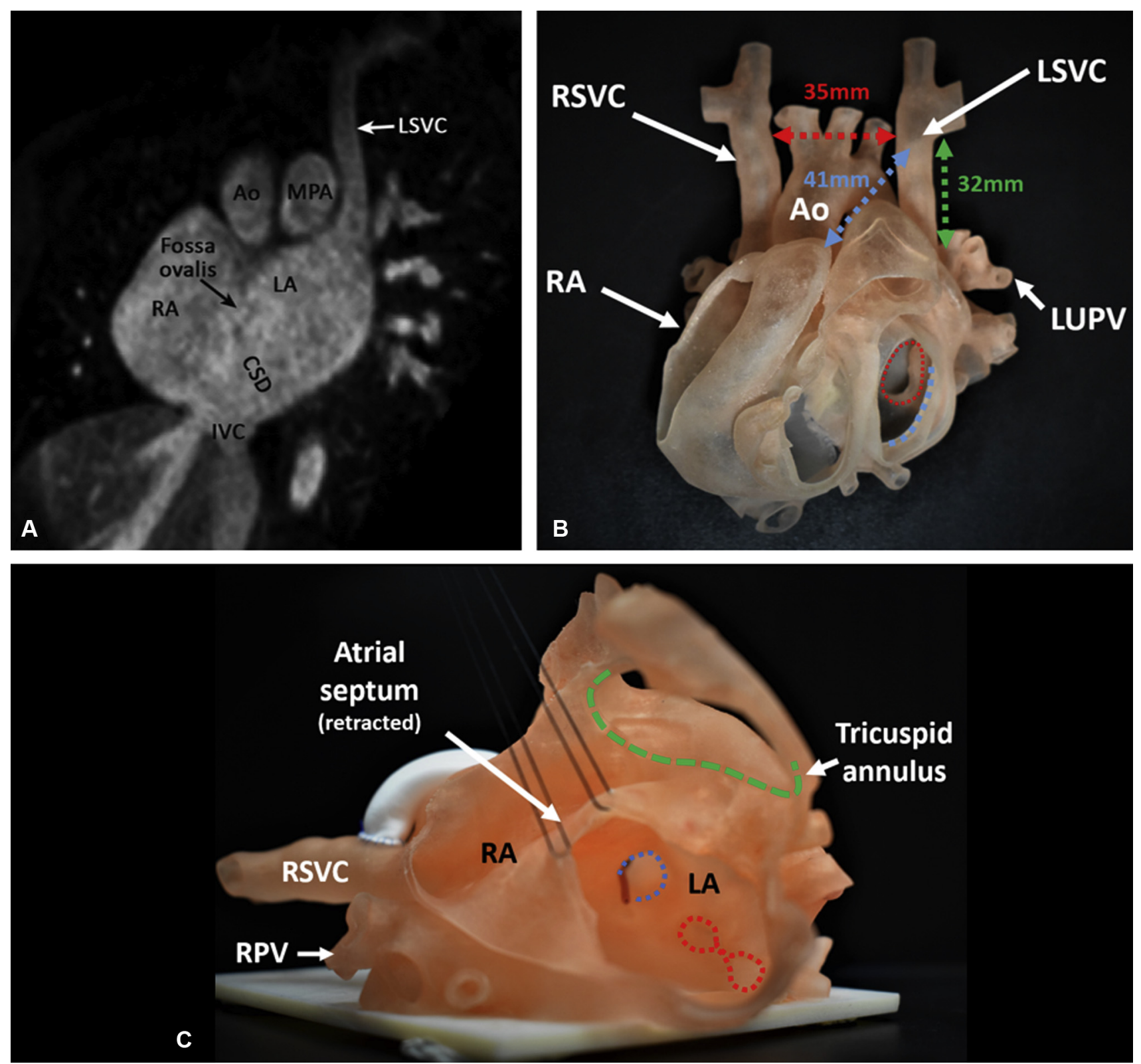

FIGURE 1. A, Reconstructed magnetic resonance image in the oblique coronal plane demonstrates the left superior vena cava ( $L S V C)$ draining direct into the left atrium $(L A)$ and a large coronary sinus defect $(C S D)$ in the lower most part of the atrial septum. B, Three-dimensionally printed model demonstrates the patient's complex anatomy. The ventricles have been removed below the level of the atrioventricular junction. Measurements were made on the model to assess feasibility of extracardiac repair. Red arrow indicates the distance between the left superior vena cava ( $L S V C)$ and the right superior vena cava $(R S V C)$; green arrow indicates the dissectible length of the left superior vena cava; blue arrow indicates the distance between the left superior vena cava and the right atrial appendage; blue dashed circle indicates the mitral annulus; red dashed circle indicates the orifices of pulmonary veins. C, Three-dimensionally printed model demonstrating the left superior vena cava $(L S V C)$ connecting to the left atrium $(L A)$ and a large coronary sinus defect. The atrial septum has been retracted to show the pathway from the left superior vena caval orifice to the right atrium $(R A)$. A small probe was passed through the left superior vena caval orifice to the left atrium. Blue dashed circle indicates the left superior vena caval orifice; red dashed circles indicate the orifices of left pulmonary veins. Ao, Aorta; $M P A$, main pulmonary artery; $R A$, right atrium; $I V C$, inferior vena cava; $L U P V$, left upper pulmonary vein; $R P V$, right pulmonary vein.

mitral inflow. Extracardiac repair was not pursued because of the possible risk of anastomotic stenosis and the minimal distance between the ascending aorta and sternum seen on computed tomography. The baffles were completed with autologous pericardium. Total cardiopulmonary bypass times were 120 and 125 minutes, with aortic crossclamp times of 88 and 83 minutes. Postoperative echocardiograms showed no residual interatrial shunt, no baffle or LSVC obstruction, and no flow acceleration across the mitral valve. There were no operative complications, and both patients were discharged within 5 days. After 5 months of follow-up, both patients remain well. The intracardiac anatomy was 

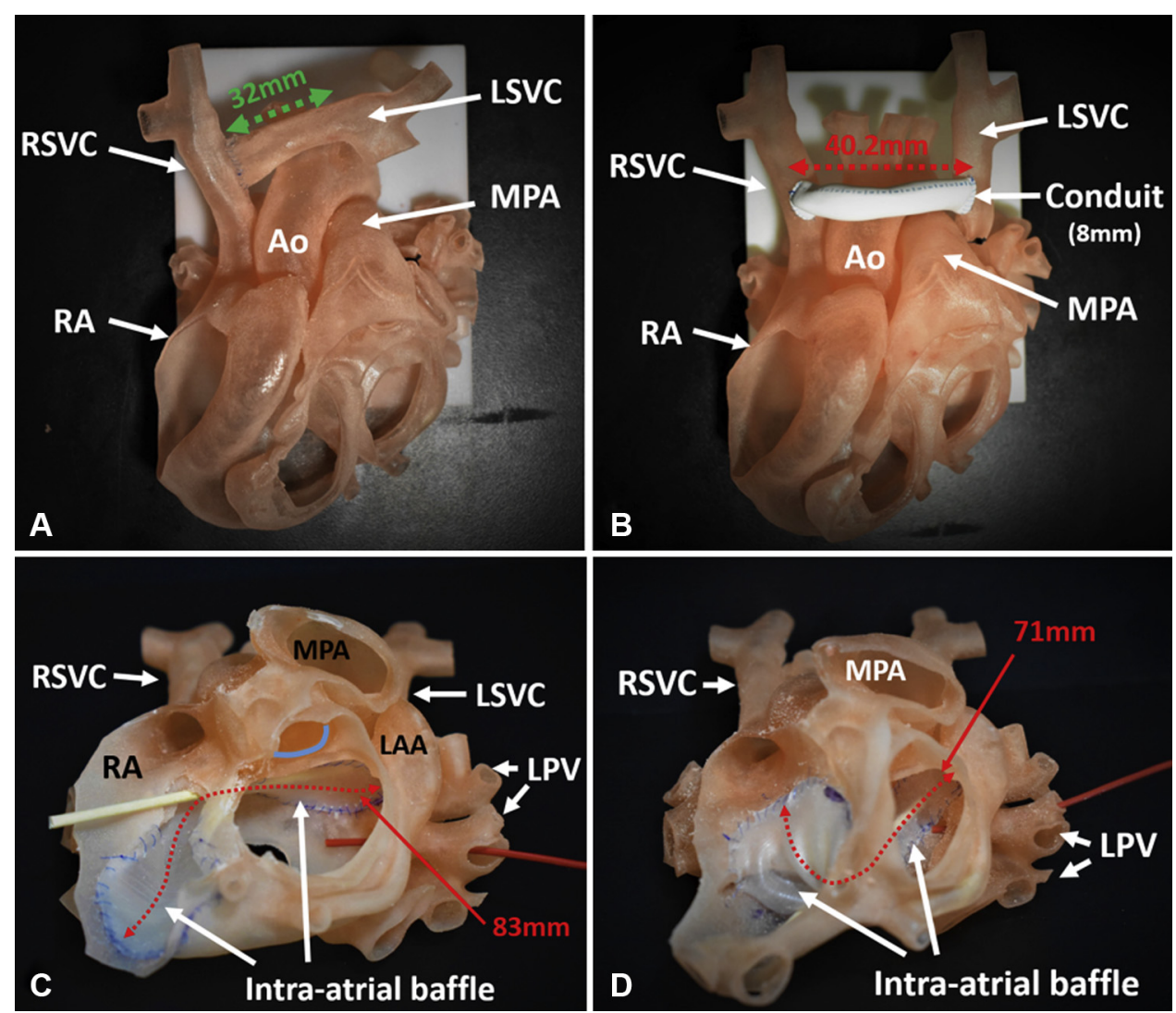

FIGURE 2. Repairs simulated on the 3-dimensionally printed models. A, Transection of the left superior vena cava $(L S V C)$ at its orifice with the left atrium and direct anastomosis with the right superior vena cava $(R S V C)$. Green arrow indicates the length of the dissected left superior vena cava. B, Ligation of the left superior vena cava $(L S V C)$ at its orifice and anastomosis with the right superior vena cava $(R S V C)$ through an 8-mm conduit. Red arrow indicates the length of the conduit. $\mathrm{C}$, Intra-atrial baffle from the left superior vena caval $(L S V C)$ orifice to the right atrium $(R A)$ along the roof of the left atrium. Beige probe shows baffle patency; red probe through the left pulmonary vein $(L P V)$ shows adequate distance from the pulmonary venous orifice; red dashed arrow indicates length of the patch $(83 \mathrm{~mm})$; blue line indicates aortic annulus. D, Intra-atrial baffle from the left superior vena caval orifice to the right atrium along the floor of the left atrium. Beige probe shows baffle patency. Red probe through the left pulmonary vein ( $L P V)$ shows the close proximity of the baffle to the pulmonary venous orifice. Red dashed arrow indicates the measured length of the patch (71 mm). Ao, Aorta; MPA, main pulmonary artery; $R A$, right atrium; $L A A$, left atrial appendage.

accurately portrayed in both models and assisted the surgeon. The major benefit was the reduced time for decision making during the operations.

\section{DISCUSSION}

Raghib syndrome results in a large left-to-right shunt through the coronary sinus defect and arterial desaturation, with a risk of paradoxical embolism, as a result of the connection of the LSVC to the left atrium. Transthoracic echocardiography provides an adequate diagnosis of Raghib syndrome, whereas cross-sectional imaging allows clearer definition of the surgical anatomy. In turn, 3Dprinted models provide surgeons with an opportunity to plan and practice the intended procedure.

The use of intracardiac and extracardiac repairs is well documented. $^{2-4}$ Concerns of the intracardiac repairs include pulmonary or mitral inflow obstruction and the risk of arrythmias. ${ }^{3,4}$ Of the extracardiac repair methods, direct anastomosis is preferred because it maintains growth potential and has lower thrombotic complications than synthetic grafts; however, the LSVC has to be long enough to avoid any tension on the anastomosis. ${ }^{2}$ Techniques include anastomosing to the RAA, tunneling through the transverse sinus to the RSVC, and creating a bidirectional left superior cavopulmonary anastomosis with the pulmonary artery. ${ }^{3}$ The use of a conduit is technically easier, because it does not require crossclamping and avoids thorough dissection of the LSVC, removing the risk of phrenic nerve injury. ${ }^{2}$ In addition to deciding what method was best from the models, it was planned that extension of the atrial septal defect superiorly was possible, followed by the singlepatch technique to create the baffle and simultaneously close the atrial septal defect.

The current surgical simulation with 3D-printed models is limited by its inability to assess the results of the repair objectively in a dynamic setting. Furthermore, the benefit of preoperative surgical simulation in patients with 
systemic or pulmonary venous abnormalities should be assessed in a larger cohort.

\section{CONCLUSIONS}

This report highlights the successful use of 3D printing in the simulation and presurgical planning in patients with Raghib syndrome.

The Cardiac 3D Printing Program at the Hospital for Sick Children is generously supported through the work of Mr Peter Butler (TOF 1959) and Mrs Fabiola Butler.

\section{References}

1. Raghib G, Ruttenberg HD, Anderson RC, Amplatz K, Adams P, Edwards J. Termination of left superior vena cava in left atrium, atrial septal defect and absence of coronary sinus; a developmental complex. Circulation. 1965;31: 906-18.

2. Ak K, Hamidov A, Ileri C, Tigen K, Isbir S, Arsan S. Correction of cortriatriatum sinister with classical Raghib's complex using an extracardiac conduit. J Card Surg. 2017;32:729-31.

3. Reddy VM, McElhinney DB, Hanley FL. Correction of left superior vena cava draining to the left atrium using extracardiac techniques. Ann Thorac Surg. 1997;63:1800-2.

4. Pérez-Caballero R, Plata Izquierdo B, Gil-Jaurena JM. Raghib syndrome. Surgical treatment. Rev Esp Cardiol. 2016;69:71. 